\title{
Seguridad Social brasileña y Trabajo Social: consideraciones sobre el Beneficio de Prestación Continua $^{1}$
}

\author{
Brazilian Social Segurity and Social Work: reflections on the Continuous \\ Cash Benefit Programme
}

\author{
Roberta STOPA \\ Pontificia Universidade Católica de São Paulo, Brasil \\ stoparoberta@gmail.com
}

Recibido: $18 / 09 / 2015$

Revisado: $14 / 11 / 2015$

Aceptado: 06/04/2016

Disponible on line: 01/07/2016

\begin{abstract}
Resumen
La seguridad social brasileña, garantizada por la Constitución federal de 1988, comprende los derechos relativos a la salud, previsión social y asistencia social. El Beneficio de Prestación Continua (BPC) fue aprobado en los objetivos de la política de asistencia social y está regulado por la Ley Orgánica de Asistencia Social de 1993. Esta prestación garantiza un salario mínimo mensual a las personas con discapacidad y a las personas mayores. Para acceder a este beneficio los requisitos que hay que cumplir son: la evaluación médica y social de la persona con discapacidad, la edad de 65 años para la persona mayor, y para ambos, la renta per capita del núcleo familiar, cuyo valor ha de ser inferior a la cuarta parte del salario mínimo. Con la regulación del BPC se han producido avances y retrocesos en la legislación y en su ejecución, de ahí que este artículo se propone presentar: una reflexión teórica, el análisis de la legislación sobre la temática y algunas consideraciones sobre los desafíos que se plantean para los trabajadores sociales.
\end{abstract}

Palabras clave: Seguridad Social, asistencia social, previsión social, Beneficio de Prestación Continua, Trabajo Social.

\begin{abstract}
Guaranteed under the Federal Constitution of 1988, Brazilian social security covers rights relating to health, social welfare and social care. The Continuous Cash Benefit Programme (BPC) was approved as part of social care policy and is regulated under the Social Care Act (Ley Orgánica de Asistencia Social) of 1993. This benefit guarantees a minimum monthly income for persons with disabilities and for older adults. Certain requirements must be satisfied in order to obtain the assistance: medical and social assessment of disabled persons, a minimum age of 65 years for older adults, and, in both cases, the value of per capita income for the nuclear family in question, which must be lower than a quarter of the minimum wage. Regulation of the BPC has incorporated advances and setbacks in terms of legislation and implementation. In this framework, this article presents a theoretical reflection, an analysis of the legislation on the matter, and some reflections on the challenges that it poses for social workers.
\end{abstract}

Keywords: Social Security, social care, social welfare, Continuous Cash Benefit Programme, Social Work.

Referencia normalizada: Stopa, R. (2016): «Seguridad Social brasileña y Trabajo Social: consideraciones sobre el Beneficio de Prestación Continua». Cuadernos de Trabajo Social, 29(2): 323-333.

Sumario: Introducción. 1. La seguridad social brasileña posterior a 1988. 2. La política de asistencia social y el Beneficio de Prestación Continua. 3. El Trabajo Social en el Instituto Nacional del Seguro Social de Brasil: los desafíos de siempre y los actuales. 4. Conclusiones. 5. Referencias bibliográficas.

${ }^{1}$ Este artículo es fruto de reflexiones hechas a partir del proyecto de investigación del Doctorado en Trabajo Social, y fue escrito durante el período de beca financiada por la Coordenação de Aperfeiçoamento de Pessoal (CAPES Brasil), en la Universitat Autònoma de Barcelona, España. 


\section{Introducción}

Brasil, aunque esté desarrollado económicamente, es un país que mantiene una gran desigualdad social y una de las peores distribuciones de riqueza y renta del mundo ${ }^{2}$. Esta concentración es una de las características más destacadas de la historia del país, y las legislaciones sociales, a pesar de ser un medio para la modificación de esta realidad, no alteran esta estructura.

Para analizar la situación de la seguridad social brasileña actual es esencial retomar lo que fue el proceso de redemocratización en los años 1980, y lo que efectivamente significó para las políticas sociales. Después de un largo periodo de régimen militar, hubo la apertura democrática y el fortalecimiento de los movimientos sociales, y muchas discusiones y movimientos fueron dirigidos a la Asamblea Nacional Constituyente, lo que se reflejó en la Constitución Federal de 1988 , fruto de las reivindicaciones y grupos organizados por sindicatos, asociaciones y partidos políticos, etc.

La nueva Constitución presentó avances en lo que respectaba a las políticas sociales y tuvo una gran participación de la población en su formulación y aprobación. Por primera vez la seguridad social se garantizaba en el país en un texto Constitucional, la cual: «comprende un conjunto integrado de acciones de iniciativa de los Poderes Públicos y de la sociedad con el objetivo de asegurar los derechos relativos a la salud, a la previsión social y a la asistencia social» (1988, p. $33)^{3}$. Desde ese momento se amplió la concepción de protección social más allá del sistema contributivo.

Es decir, la seguridad social significa la cobertura de estas tres políticas, conformando así un sistema de protección social. La salud se garantiza a todas las personas sin cotización previa, la previsión social depende de cotizaciones previas y la asistencia social tiene carácter selectivo, ya que garantiza el derecho a quien la necesita; según Boschetti (2004) conjuga derechos con carácter universal (salud), con derechos derivados del trabajo (previsión) y derechos selectivos (asistencia social).

Sin embargo, este marco histórico acontece en un contexto mundial de crisis y de restructuración de los derechos sociales en una perspectiva neoliberal, por lo tanto, inmediatamente después de la aprobación de la denominada Carta Ciudadana se empieza a incorporar las medidas de esta orientación.

La asistencia social pasó por un proceso histórico en el ámbito de la caridad y de la filantropía, por medio de las acciones denominadas asistenciales se intentaba paliar la pobreza, o mejor, la contención de las manifestaciones de la cuestión social ${ }^{4}$.

Aun con los avances, fundamentalmente en el ámbito jurídico, todavía es necesario superar la cultura de la ayuda y de la filantropía. Es un reto para la política de asistencia social consolidarse en el campo, un tanto árido en Brasil, de los derechos sociales, y volverse efectivo bajo los principios constitucionales de la democracia participativa y de la descentralización.

En el artículo 203 de la Constitución se estableció, como uno de los objetivos de la asistencia social, la garantía de un salario mínimo mensual a la persona mayor y a la persona con discapacidad que prueben que no tienen medios para su propio mantenimiento y ni de que lo pueda realizar su familia. Este beneficio se reguló con el denominado como Beneficio de Prestación Continua (BPC) por medio de la Ley orgánica de asistencia social (LOAS, 1993).

Aunque el beneficio se gestione y financie por la asistencia social, el Beneficio de Prestación Continua lo maneja el Instituto Nacional del Seguro Social (INSS), órgano responsable por la política de previsión social, imprimiendo numerosos y complejos desafíos en la garantía de esta prestación. De esta manera, es necesario evaluar el Beneficio de Prestación Continua en el campo de la seguridad social, en el actual con-

\footnotetext{
${ }^{2}$ Según Ávila (2014): «Los 0,9\% más ricos del país poseen entre 59,90\% y 68,49\% de la riqueza».

${ }_{3}$ Todas las citas de este artículo fueron traducidas por la autora, toda la referencia bibliográfica está en portugués.

${ }^{4}$ Según Iamamoto (2004), cuestión social es el conjunto de las expresiones de la desigualdad social, propia de la sociedad capitalista. «La producción social es cada vez más colectiva, el trabajo se vuelve más ampliamente social, mientras la apropiación de los frutos permanezca privada, monopolizada por una parte de la sociedad» (p. 27).
} 
texto de la contrarreforma ${ }^{5}$ del Estado brasileño y de la deconstrucción de las políticas sociales.

La relación del Trabajo Social con las políticas sociales es de larga duración; sea en la intervención profesional, en los análisis y producciones teóricas, o incluso, por la participación en los movimientos de defensa de los derechos sociales, muchos profesionales participaron en la lucha por la garantía de las políticas de seguridad social en el proceso constitucional. Sin embargo, la política de asistencia social es un locus privilegiado de trabajo del asistente social, además, es un tema importante investigado y analizado por los profesionales, ofreciendo una base teórica para su formulación, implementación y ejecución. Pero esto no significa que el Trabajo Social se limite a esta política.

Por lo tanto, el objetivo de este artículo es discutir sobre los avances y los retrocesos en la legislación brasileña sobre el Beneficio de Prestación Continua y los retos en el trabajo cotidiano de los asistentes sociales. Así, se presenta una breve contextualización de la seguridad social brasileña, de la política de asistencia social y del Beneficio de Prestación Continua; y, posteriormente sobre el Trabajo Social y su inserción y actuación en el Instituto Nacional del Seguro Social, y una reflexión sobre los desafíos frente a los retrocesos y los avances en la legislación brasileña.

\section{La seguridad social brasileña posterior a 1988}

Para la aprobación de la seguridad social fueron necesarias numerosos debates. Hubo propuestas conservadoras e innovadoras, se mantuvieron las proposiciones de ambas partes con gran avance en lo que se refiere a los derechos sociales.

Al principio de la década de 1990, bajo la influencia de la ideología neoliberal, se iniciaron los recortes relacionadas con los seguros sociales de los asegurados, y para otras políticas. Por lo tanto, a pesar del reconocimiento legal, el proceso de transformar las prerrogativas constitucionales en derechos no ocurrió como se había prescrito (Behring y Boschetti, 2008). Se puede citar como ejemplo el retraso en la aprobación de las leyes orgánicas y la no formación del Consejo de Seguridad Social, entre otros aspectos que se habían previsto.

Las tres políticas de seguridad social se vieron afectadas directamente por la contrarreforma del Estado, favoreciendo al sector privado en detrimento del público. Siempre con el argumento de la reducción de los costes, con clara indicación de la falta de la democratización de la gestión de la seguridad social. «En esta nueva arquitectura institucional, por lo tanto, el ciudadano de derechos se convierte en ciudadano-cliente, consumidor de servicios de organizaciones cuyo comportamiento está guiado por la perspectiva empresarial sujeta a la presentación de resultados» (Behring, 2008, p. 259).

Es importante destacar que la seguridad social asegura la protección social, mientras tanto, además de la alta tasa de trabajo informal ${ }^{6} \mathrm{y}$ de desempleo, el capital prioriza la reducción de los derechos sociales, conquista de los trabajadores que muchas veces son presentadas como privilegios, y la reducción de las garantías laborales en forma de flexibilización.

La flexibilización del trabajo consiste en la reducción de costes y el aumento del lucro, con lo que comporta de disminución en los salarios. En esta situación se inserta el trabajador «polivalente» $\mathrm{y}$ hay una reducción del número de trabajadores tanto en el sector privado como en el público. El desarrollo científico y la reestructuración productiva afectan a la producción de bienes y servicios, la organización, la gestión y las condiciones y relaciones de trabajo.

En el capitalismo contemporáneo hay una diminución de la clase obrera tradicional, pero al mismo tiempo crece el número de trabajadores en el sector de servicios y también la subproletarización que, según Antunes (2008), es la «[...] expansión del trabajo a tiempo parcial, temporal, precario, subcontratado, «tercerizado», que marca la sociedad dual en el capitalismo avanzado»» (p. 47). El resultado es el desempleo masivo, y no hay una tendencia única, sino lo que el autor denomina «un proceso de mayor heterogeneidad,

${ }^{5}$ Behring (2008) hizo un estudio de la reforma del Estado durante el gobierno de Fernando Henrique Cardoso. La autora lo denomina de contrarreforma del Estado «[...] que implicó un profundo retroceso social en beneficio de pocos» (p. 22).

${ }^{6}$ Según Boschetti (2009), sólo el 47,8\% de los trabajadores son formales, es decir, afiliados a la seguridad social. 
fragmentación $y$ complejidad de la clase trabajadora» (p. 47).

En Brasil las reformas en las políticas de seguridad social no eliminarán el sistema público y tampoco el modelo de contribución, sino que modifican el alcance y la estructura de los beneficios (Boschetti, 2009). Con los cambios en el mundo del trabajo y con los ataques a las políticas de seguridad social, así como la inconclusión de las garantías constitucionales, y con el poco compromiso por parte del gobierno, la garantía de los derechos sociales se constituye en un campo interminable de lucha para la clase trabajadora.

\section{La política de asistencia social y el Benefi- cio de Prestación Continua}

En el proceso de definición de la seguridad social la presión popular fue de extrema importancia, pero la asistencia social necesitaba mayor presión, puesto que ocurrieron pocas reivindicaciones en relación con la reglamentación de la política. Así la asistencia social fue la política que tuvo más contratiempos para la aprobación de su reglamentación - la salud fue reglamentada por la Ley 8.080 de 1990; y la previdencia social por la Ley 8.213 en 1991 - y ocurrió solamente en 1993, con la Ley 8.742 (LOAS).

A pesar de las dificultades y los retrocesos durante estos años hubo muchos debates, sobre todo en los espacios de las conferencias y los consejos, y también se aprobaron normas y nuevas leyes con numerosos avances, aunque tímidos e insuficientes para garantizar lo previsto en la Constitución Federal de 1988. Entre estos avances se pueden destacar: la aprobación de la Política Nacional de Asistencia Social (PNAS) en 2004 y del Sistema Único de Asistencia Social (SUAS) ${ }^{7}$ en 2005.

La Política Nacional de Asistencia Social de 2004 asegura la implantación del Centro de Referencia de Asistencia Social (CRAS) en los municipios, que es una unidad pública estatal de ba- se territorial, ubicado en zonas de vulnerabilidad social, y presta servicios de protección social básica; organiza y coordina la red de servicios sociales locales de la política de asistencia social. También la implantación del Centro de Referencia Especializado de Asistencia Social (CREAS), que es la unidad pública de cobertura y gestión municipal, estadual o regional, destinada a la prestación de servicios a individuos y familias que están en situación de riesgo personal o social, por violación de derechos que requieren intervención especializada de la protección social.

La implantación de los Centros es una de las innovaciones introducidas por la política, ya que en muchas ciudades brasileñas no había una estructura pública para la intervención efectiva de la asistencia social. Es evidente que hubo muchos obstáculos y dificultades financieras y administrativas para la implantación de esos Centros. Además el Sistema Único de Asistencia Social fue reglamentado solamente en 2011, con la aprobación de la Ley 12.435.

En la Ley orgánica de asistencia social se definieron los criterios para el acceso al Beneficio de Prestación Continua, pero su implantación se produjo sólo en 1996, por el Decreto 1.744 de 8 de diciembre de 1995, es decir, ocho años después de la aprobación de la Constitución Federal.

El Beneficio se atribuye a las personas mayores y a las personas con discapacidad, y la renta per capita de la familia tiene que corresponder al valor inferior a la cuarta parte del salario míni$\mathrm{mo}^{8}$. Estos criterios, a pesar de haber sufrido algunas modificaciones a lo largo de los años, siguen aún vigentes.

En relación al Beneficio ${ }^{9}$ para la persona mayor, el art. $20^{\circ}$ de la Ley orgánica de asistencia social garantiza el acceso a la persona con 70 años o más, esa edad debía ser reducida a los 67 años a los 24 meses de la aprobación de la Ley, y para 65 años después de más 24 meses. Pero el Decreto 9.720 de 1998 estableció que la edad pa-

${ }^{7}$ El sistema unificado es un modelo de gestión para todo territorio nacional que articula acciones de los tres entes federativos para la asistencia social. Este Sistema tiene como objetivo regular y organizar los servicios socioasistenciales.

${ }^{8}$ El salario mínimo en 2015 es de 788,00 reales, la cuarta parte de este valor son 197,00 reales. En dólares, el valor para tener derecho al Beneficio de Prestación Continua son 55,64 dólares americanos. Las cifras corresponden a la cotización del día 24 de agosto de 2015.

9 Según los datos del Ministerio del Desarrollo Social (MDS) actualmente hay 3.600 .000 beneficiarios, de este total 1.900 .000 son personas con discapacidad y 1.700 .000 son personas mayores. Disponible en: http://www.mds.gov.br/assistenciasocial/beneficiosassistenciais/bpc/beneficio-de-prestacao-continuada-bpc 
ra acceder al Beneficio, a partir de ese año serían los 67 años. La reducción a los 65 años ocurrió sólo en 2003 con el «Estatuto del Anciano».

El acceso al Beneficio para la persona con discapacidad lo garantizó la Ley orgánica de Asistencia social de 1993 de la siguiente manera: Art. 20 párrafo $2^{\circ}$ : «Para efecto de concesión de este beneficio, la persona con discapacidad es aquella incapacitada para la vida independiente y para el trabajo». Durante algunos años la evaluación de la discapacidad se llevó a cabo por médicos del Sistema Único de Salud (SUS) y, posteriormente, pasó a ser realizada por los peritos médicos del Instituto Nacional del Seguro Social.

El Decreto 6.214, aprobado en 2007, introdujo la evaluación social y médica con base en la Clasificación Internacional de Funcionalidad, Incapacidad y Salud (CIF). Sin embargo, aún siguió siendo el concepto de incapacidad para el trabajo, según el art. $9^{\circ}$ : «Para hacer justicia al Beneficio de Prestación Continua la persona con discapacidad deberá comprobar: I - ser incapaz para la vida independiente y para el trabajo» (p. 3).

Incluso con la aprobación de la ley 12.435 del 6 de julio de 2011 —que dispone sobre la organización de la asistencia social- no hubo alteración. Sólo la ley 12.470, aprobada en 31 de agosto de 2011, alteró la comprensión de discapacidad al eliminar el término «incapacidad para la vida independiente y para el trabajo»».

En 2011, se incluyó una nueva pregunta en la evaluación médica relativa a la discapacidad, sobre impedimentos de largo plazo (2 años), aunque en las evaluaciones se haya constatado que la persona tiene impedimentos de carácter físico, mental, intelectual o sensorial, el beneficio no será concedido si el perito médico indica que no haya impedimento de largo plazo.

Esto ya había sido establecido por la Ley orgánica de Asistencia social, cuando aseguraba la revisión bienal ${ }^{10}$ del Beneficio. O sea, que cada dos años es necesario hacer la revisión, pero lleva algunos años que no se ha realizado, y con la introducción de esta pregunta en la evaluación médica se puede pensar que tampoco se harán muchas revisiones.
Para el acceso al Beneficio de Prestación Continua, que es un beneficio asistencial, no se exige una aportación directa, pero la mayoría de las personas que solicitan este beneficio han ejercido en algún momento de su vida actividades laborales, aunque para el acceso al beneficio haya necesidad de no estar insertado en el mercado de trabajo e incluso su incapacidad para ello. Sobre esta situación, Silva (2012) explica que: «Las personas con alguna discapacidad, aunque no limite su capacidad de trabajo, y las personas mayores con edad de 65 años, desempleadas o sin condiciones de continuar contribuyendo con la previsión, buscan el acceso al BPC como alternativa» (p. 394).

Masson (2011), en su investigación sobre los impactos del Beneficio de prestación continua, identificó que muchos beneficiarios además de trabajar en algún período de sus vidas y haber contribuido directamente a la previsión social, pero no lo han hecho lo suficiente como para asegurar la jubilación. De los beneficiarios entrevistados el 68,83 por ciento informó que habían trabajado y el 30,18 por ciento que habían cotizado al régimen general de la previsión social ${ }^{11}$ cerca de 9 años.

En relación a los beneficiarios de esta prestación, por los datos de la investigación llevada a cabo por Masson (2011), el beneficio es utilizado para cobertura de las necesidades humanas: comer, vivir, vestirse, etc. "Satisfacer la necesidad de alimentarse a través del BPC fue destacado por $97,4 \%$ de los entrevistados, los beneficiarios, también usan el beneficio en la medida de lo posible en la salud, así como, la vestimenta» (p. 95).

Este hecho remite a la cuestión de cómo tienen sus necesidades humanas satisfechas las personas mayores y las personas con discapacidad, que no estén insertadas en el mercado de trabajo y no tienen acceso al Beneficio debido a los estrictos criterios. Es importante reflexionar que, aunque tengan el beneficio, esas necesidades no son satisfechas, ya que las condiciones de vida de las personas son marcadas por la ausencia o la exclusión de acceso a los derechos.

A pesar del gobierno, lanzar programas de combate a la pobreza extrema - por ejemplo, el

10 «Art. 21. El beneficio de prestación continua debe ser revisado cada 2 (dos) años para evaluación de la continuidad de las condiciones que dieron origen» (Ley 12.435, 2011, p. 3).

11 Actualmente para la jubilación por edad es necesario haber cotizado 15 años, la edad de 60 años para la mujer y de 65 años para el hombre. 
Programa Bolsa Familia y el Plano Brasil sin Miseria - el acceso al Beneficio, única prestación de la asistencia social con valor de salario mínimo, es muy estricto, ya que el criterio de renta y el concepto de familia deja a millares de personas sin posibilidad de acceso al Beneficio ${ }^{12}$.

El concepto de familia fue modificado a partir de la citada Ley $12.435 / 11$, donde se considera como componentes de aquélla, de cara al cálculo de la renta, a los hermanos e hijos solteros, mayores de 21 años, y considera al padre y la madre, incluso cuando la persona es mayor de 21 años, lo que hace aún más difícil el acceso al beneficio. En el art. 20 párrafo 1ํ la familia está compuesta por: «solicitante, el cónyuge o compañero, los padres $\mathrm{y}$, a falta de uno de ellos, la madrasta o el padrastro, los hermanos solteros, los hijos e hijastros solteros y los menores tutelados, a condición de que vivan bajo el mismo techo» (p. 11).

En otras palabras, con el SUAS el campo de actuación de la asistencia social fue ampliado, pero el concepto de familia y el recorte de renta del BPC aún limitan la garantía de acceso de los ciudadanos a esa política.

Aunque el Beneficio de Prestación Continua sea una prestación asistencial, lo gestiona el Instituto Nacional del Seguro Social porque, según Maciel (2008), la asistencia social no tiene estructura para ese trabajo. Sin embargo esta situación ha ido cambiando a lo largo de los años, principalmente después de la aprobación del Sistema Único de Asistencia Social en 2005, ya este Instituto tiene estructura en todo el país para conceder este Beneficio. De ahí que, por hallarse en el Instituto Nacional del Seguro Social, se comprende, justamente, que el Beneficio es una suerte de jubilación de la previsión social. Maciel (2008) destaca que:

Es muy probable que el BPC, por ser gestionado por el INSS, sea identificado y reconocido como un beneficio de la previsión por la población demandante. Es porque el contexto del INSS tiende a irradiar el reflejo de su identidad simbólica sobre el BPC, lo que ocasiona una percepción errónea del beneficio asistencial (p. 106).

Para solicitar el Beneficio, el ciudadano tiene que presentar en el Instituto la solicitud y los do- cumentos personales del solicitante y de las personas que componen la familia. El Beneficio tiene carácter declarativo, pero a menudo los prestadores hacen peticiones excesivas, lo que dificulta el acceso a la prestación, ya que con tantas restricciones, como por ejemplo, la de exigir la sentencia del divorcio para comprobar que el solicitante no recibe la pensión alimenticia, aunque la persona haya declarado que no la percibe. Maciel (2008) en su investigación comprobó que: «la tendencia expuesta es que el anclaje institucional promovido por el INSS otorga la "naturalización" de los sistemas de clasificación y de evaluación expuesta en las manifestaciones de los servidores de la institución» (p. 144).

La cotidianidad institucional reproduce la idea de seguro, y de que sólo los que trabajan formalmente o los que contribuyen a la previsión social pueden tener acceso a los beneficios. Esa situación desplaza los principios éticos del derecho a la asistencia social: «debido a la imposición de criterios y requisitos técnicos ajustados a la lógica que la demostración, distinta de la lógica de la asistencia social como política pública de seguridad social no contributiva»» (p. 149).

Después de otorgarle el beneficio, la persona con discapacidad pasa por la evaluación social y médica. Para la realización de la evaluación social es necesario que el Instituto Nacional del Seguro Social tenga en su plantilla de profesionales a trabajadores sociales, pero hacía años que no había un concurso público para su contratación - había cerca de 450 profesionales en todo Brasil - lo que estaba reivindicándose hacía tiempo. El concurso tuvo lugar en 2008 y las contrataciones de los profesionales en 2009.

\section{EI Trabajo Social en el Instituto Nacional del Seguro Social: los desafíos de siempre y los actuales}

En Brasil, de acuerdo con Iamamoto (2007): «es de suma importancia el trabajo que los asistentes sociales están realizando especialmente en el ámbito de la seguridad social: en los procesos de su elaboración, gestión, supervisión y evaluación en los distintos niveles de la federación» (p. 198).

El surgimiento de la profesión está vinculado a los proyectos políticos y económicos que reproducen material e ideológicamente la hegemo-

${ }^{12}$ Entre los años de 2008 a 2012 fueron denegados por renta 457.707 solicitudes, siendo 243.484 por persona con discapacidad y 214.223 por personas mayores (Vaz, 2013). 
nía de la clase dominante en el capitalismo, en el cual el Estado asume las respuestas a la cuestión social. Sobre eso Yazbek (2009) explica:

Analizar el Trabajo Social en esta perspectiva permite, en primer lugar, aprehender las implicaciones políticas del trabajo profesional que se desarrolla en el contexto de las relaciones entre las clases. Es decir, comprehender que la práctica profesional del Trabajo Social es necesariamente polarizada por los intereses de las clases sociales en esa relación, no se puede pensar en ella fuera de esa trama. Permite también aprehender las dimensiones objetivas y subjetivas del trabajo del asistente social (p. 128).

Es a partir del año 1980 cuando la profesión empieza a trazar una interlocución con la teoría marxista. Ese proceso de introducción de nuevos referentes teórico-metodológicos y de intervención se expresa en los debates y producciones científicas de la profesión; resultado de una amplia producción teórica del Trabajo Social. Además el inicio de los cursos de posgrado stricto sensu en la década de 1970 aumentó el número de profesionales cualificados para la docencia y la investigación.

A pesar de los cambios que experimentó el Trabajo Social brasileño, en los años 1980 y 1990, que se manifestaron en el intento de romper con el conservadurismo, aún está presente «la herencia conservadora y antimoderna» (Yazbek, 2009). Sobre la madurez profesional, la autora explica que: «avanza en el inicio del nuevo milenio que se expresa por la democratización de la convivencia de los diferentes posicionamientos teórico-metodológicos e ideopolíticos desde finales da década de 1980» (p. 11).

El Trabajo Social está regulado como una profesión liberal con estatutos jurídicos y éticos ${ }^{13}$ que garantizan una autonomía teórico-metodológica, técnica y ético-política pero, como explica Iamamoto (2004), esa autonomía es relativa, ya que el asistente social vende su fuerza de trabajo especializada a los empleadores; o sea, es un trabajador asalariado. Así el trabajo profesional es apropiado por otro, y ese determina las necesidades sociales a las que debe responder el trabajo.

La intervención del Trabajo Social se basa en la realidad social. El surgimiento, el desarrollo y la ocupación de un espacio en la división sociotécnica del trabajo son «resultantes de las relaciones históricas, sociales, políticas, económicas y culturales que moldean su necesidad social, estructuran sus estrategias de intervención y definen los usuarios» (Yazbek, 2008, p. 119).

Según Netto (2009), en el proceso de enfrentamiento de la profesión al conservadurismo están las bases del proyecto profesional nuevo, llamado Proyecto Ético-político, que es el resultado de un largo proceso colectivo construido por los trabajadores sociales, teniendo al frente las entidades nacionales: Consejo Federal de Servicio Social (CFESS), Consejos Regionales de Servicio Social (CRESS), Asociación Brasileña de Enseñanza e Investigación en Servicio Social (ABEPSS) y Ejecutiva Nacional de los Estudiantes de Servicio Social (ENESSO).

El Proyecto pasa a tener un carácter hegemónico para la profesión a partir de la década de 1990. Sin embargo no significa que se lo hayan apropiado los trabajadores, ya que ese movimiento, per se, no eliminó las tendencias conservadoras presentes en la profesión. Netto (2009) explica que el Proyecto tiene como valor central el reconocimiento de la libertad - concebida históricamente como posibilidad de elección entre alternativas concretas.

Por lo tanto, este Proyecto profesional se vincula a un proyecto societario que propone la construcción de un nuevo orden social, sin explotación/dominación de clase, etnia y género [...] el Proyecto afirma la defensa intransigente de los derechos humanos y el rechazo a la arbitrariedad y al prejuicio, contemplando de manera positiva el pluralismo, tanto en la sociedad como en el trabajo profesional. (p. 155)

Es preciso destacar la importancia del Consejo Federal de Servicio Social, órgano máximo de representación de los trabajadores sociales en Brasil, como uno de los segmentos más combativos contra el conservadurismo, y su vinculación con el movimiento de los trabajadores, redimensionando ampliamente la organización profesional (Netto, 2009). Se puede destacar, como marco de este trabajo, la Ley n ${ }^{\circ} 12.317$ de 26 de agosto de 2010, que dispone sobre la duración del trabajo del asistente social: 30 horas semanales.

${ }^{13}$ Los documentos que representan los avances de la profesión son: el Código de Ética de 1993 y la Ley que reglamenta la profesión, Ley 8.662 de 1993, y en el ámbito de la formación académica las Directrices Curriculares de los Cursos del Servicio Social de 1996. 
La organización política de los trabajadores sociales es visible en los encuentros y congresos nacionales realizados para debatir diversos temas. Un ejemplo es la aprobación de la Carta de Maceió en la XIX Reunión Nacional del Consejo Federal de Servicio Social/ Consejos Regionales de Servicio Social de 2001, que representa un importante documento para el debate sobre la seguridad social. Acerca de ese documento Behring (2008) explica que los profesionales reafirmaron el compromiso con la lucha por la seguridad social pública, universal y de cualidad.

Esta defensa no acontece a partir de la concepción de la Carta, sino que es una construcción histórica y de largo proceso. El contenido de la Carta de Maceió tiene la perspectiva de superar la lógica del seguro, que es restrictiva, y avanzar en el propósito de ampliación de los derechos universales; es decir, está alineada con la dimensión política del Proyecto Ético-político del Servicio Social.

El documento refleja la comprensión de la seguridad social como un proceso histórico, en el contexto de crisis del capital, que se difunde desde la década de 1970, reconociendo los límites que se plantean por el orden económico, $\mathrm{y}$ que traza estrategias para hacerles frente.

Sobre el Trabajo Social en la previsión social, su trayectoria histórica está marcada por luchas y resistencias y por la inserción de los trabajadores sociales en los movimientos sociales y sindicales. En 1942 ocurrió la primera experiencia de implantación del Trabajo Social en la previsión social, y en octubre de 1944, con la publicación del Decreto $\mathrm{n}^{\circ} 52$ del Consejo Nacional del Trabajo, fue instituida la profesión. Los documentos que elaboraron los profesionales en el Instituto Nacional del Seguro Social - los Planes Básicos de Acción del Servicio Social (PBA) de 1972 y de 1978 y la Matriz Teórico-metodológica del Servicio Social en la Previsión Social de 1994 expresan la realidad de la profesión en su trayectoria en la institución, y muestran la lectura de la realidad que realizaron los profesionales para la construcción de estos instrumentos.
La profesión, que durante años se caracterizó por ser conservadora y tradicional, a partir de la década de 1990 empieza a crear propuestas de intervención basadas en el Proyecto Etico-político. Dos de las mayores conquistas del Trabajo Social de la previsión social fueron el artículo 88 de la Ley 8.213 de 1991, que define la competencia del Trabajo Social ${ }^{14}$, y la formulación de la ya citada Matriz Teórica Metodológica en 1994.

En la previsión social las reformas administrativas ${ }^{15}$ afectaron a la profesión, pues en 1998 se publicó la Medida provisional $n^{\circ} 1.729$, que preveía la supresión del artículo 88 de la Ley $8.213 / 91$, los trabajadores sociales se movilizaron para intentar revertir esa situación. La movilización nacional, que tuvo una amplia participación de los sindicatos, asociaciones de trabajadores y de la División del Servicio Social del Instituto Nacional del Seguro Social, tuvo su efecto, y no fue posible la extinción de la profesión. Pero, la amenaza está siempre presente en el proceso de reforma, ya que no fue la única tentativa de desmontar el Trabajo Social dentro de la previsión social. Cartaxo y Cabral (2008) comprenden el proceso como de «deconstrucción» del Trabajo Social en la previsión.

En este escenario y con la plantilla profesional con un número desfasado - casi 30 años sin concurso público - la contratación de los analistas de la seguridad social con formación en Trabajo Social representa una grande conquista:, fueron 900 los contratados, pero durante el período de validez del concurso se contrató a más profesionales. Todavía la denominación del cargo «analista» generó conflictos, la confusión entre el cargo y la función para la cual habían sido contratados los profesionales llevó - y lleva- a muchos gestores a presionar a los trabajadores sociales para que realicen actividades administrativas. Además el Instituto no cumple la Ley $12.317 / 2010$ que instituyó la carga horaria de 30 horas semanales de trabajo.

Igualmente, existen otros limites institucionales planteados a la profesión, como: la falta de

14 «Art. 88. «Es competencia del Servicio Social definir con los beneficiarios sus derechos sociales y los medios para ejercerlos y establecer conjuntamente con ellos el proceso de solución de los problemas que surgen de su relación con la Previsión Social, tanto en el ámbito interno de la institución como en la dinámica de la sociedad» (p. 33).

15 Hubo dos grandes reformas, o mejor dicho, contrarreforma en la previsión social: una en 1998 y la otra en 2003; procesos alineados a la perspectiva neoliberal que impide los avances e incluso retrocede en la garantía de los derechos sociales. 
entendimiento (o incluso de respecto) de los gestores en relación a las atribuciones de la profesión y la falta de espacio físico y de recursos materiales para la realización de los servicios. Es imprescindible que esos límites sean debatidos por los trabajadores sociales, junto con el Consejo Federal de Servicio Social/Consejos Regionales de Servicio Social y los sindicatos. Pensar en la profesión en este contexto significa, según Yazbek (2008): «tener presente que la intervención profesional del Servicio Social está condicionada por la dinámica institucional que va a configurar límites y posibilidades para la acción profesional» (p. 119).

En el Instituto Nacional del Seguro Social, los trabajadores sociales enfrentan el gran desafío de mantener el espacio de trabajo y la autonomía profesional. Hasta 2009 en muchas Gerencias Ejecutivas (órgano responsable de ejecución en el ámbito regional de la política de previsión social) no existía este profesional en su plantilla de funcionarios, entonces se inició el proceso de implantación del Trabajo Social en estos lugares. En muchas Agencias de Previsión Social (APS) no hay despacho para atender, o no es adecuado, y no hay recursos materiales necesarios para el trabajo.

Es importante destacar que el trabajador social en la previsión social actúa en diferentes proyectos, programas y sectores, como: en el Programa de Rehabilitación Profesional, en la Gestión de Personas, en la atención a los ciudadanos, etc. $\mathrm{Y}$ cuenta con dos importantes instrumentos de trabajo: el informe social y la valoración social.

El dictamen social se utiliza con el objetivo de comprobar la dependencia económica o no en los casos de pensión por muerte y auxilio por reclusión y también cuando lo solicita la Junta de Recursos en casos específicos, por ejemplo, para fines de Beneficio de Prestación Continua. A pesar de ser un importante instrumento en la garantía de derechos sociales, este recurso se utiliza poco en la Institución.

Aunque en 2007 la evaluación social ha sido garantizada por el Decreto 6.214, este trabajo solamente se inició en 2009. Las evaluaciones sociales y médicas se introducen en el sistema informatizado, la evaluación médica considera las discapacidades en las funciones y en las estructu- ras del cuerpo, y la evaluación social considera los factores ambientales, sociales y personales, y ambas consideran la limitación del desempeño de las actividades y la restricción de la participación social, según sus especificidades.

Con la introducción de la evaluación social, como ya se ha comentado, hubo que contratar a trabajadores sociales; así se intensifican los desafíos ya existentes y se introducen otros nuevos para la profesión. Uno de los desafíos es la capacitación de los nuevos profesionales y de los que ya trabajaban en la previsión. Es imprescindible el conocimiento de la CIF para la realización de este trabajo, pero el curso de capacitación de los trabajadores sociales profundizó poco en este debate. Además de la capacitación para el trabajo con los solicitantes del Beneficio de Prestación Continua, es importante el conocimiento de los beneficios de la previsión social para atender y orientar a los ciudadanos.

Otro desafío es la realización de la atención a la persona mayor y el acompañamiento a las personas sin acceso al Beneficio; en otras palabras, es necesaria la interlocución entre las políticas de previsión y de asistencia social. Está amparado por la legislación de la asistencia social el acompañamiento a esta demanda ${ }^{16}$, pero aún queda mucho que hacer para efectuar esta atención, no sólo por las dificultades administrativas y de personal, sino también por la dificultad de acceso a los datos que debería proporcionar el Ministerio del Desarrollo Social (MDS). Como esto no se logra, no se conoce el destino de millares de ciudadanos que solicitan el Beneficio y no tienen el acceso garantizado.

$\mathrm{Se}$ puede decir que es fundamental la (re)afirmación de los principios del Proyecto Ético-político para desempeñar ese trabajo, en defensa de los derechos de los ciudadanos en la atención diaria y en la realización de la valoración social como un instrumental de acceso al beneficio. Para el trabajo en la previsión social, y no solamente en esta política, es esencial comprender las contradicciones existentes en la sociedad capitalista y en el mundo del trabajo.

Entendiendo que el acceso al Beneficio de Prestación Continua depende de diversos factores, es un desafío para la profesión profundizar en el análisis de las perspectivas de la evaluación so-

16 Decreto 6.214/07 art. 41-I está garantizado: «El monitoreo de la incidencia de los beneficiarios y de los solicitantes por ciudades y en el Distrito Federal». 
cial del mismo y el estudio del instrumental, tanto por tratarse de un instrumento técnico nuevo, como por la necesidad de una reflexión constante sobre la temática. Además es necesario reflexionar sobre el Trabajo Social más allá de la valoración social, teniendo en cuenta la importancia del profesional en la atención a los ciudadanos.

\section{Conclusiones}

Bajo el trinomio privatización, selectividad y descentralización (Behring y Boschetti, 2008), la tendencia va hacia la restricción de los derechos garantizados en la Constitución Federal, transformando las políticas públicas en acciones puntuales. Las leyes que reglamentan la seguridad social no garantizaron la materialización de todos los principios previstos en la Constitución, así la seguridad social materializó políticas con características propias y específicas que, en vez de complementarse, se excluyen.

Con el avance del neoliberalismo hay un aumento de programas y proyectos selectivos y focalizados, retornando a la escena prácticas filantrópicas y voluntaristas, lejos del principio de universalización de los derechos sociales. El Trabajo Social está inserido en este contexto contra- dictorio, es importante que los profesionales reflexionen sobre esta realidad y tracen estrategias de intervención.

Frente a las dificultades al acceso al Beneficio de Prestación Continua y a la comprensión del mismo como un derecho constitucional, es imprescindible que los trabajadores sociales estén capacitados para enfrentar las adversidades presentes en lo cotidiano profesional. Para el acceso de muchas personas, que tienen derecho al Beneficio conforme a la Constitución Federal de 1988, es necesario un cambio de comprensión en relación a esta prestación y a los criterios de renta, edad, composición familiar y concepto de discapacidad; este último ya está cambiado por la legislación, pero necesita ser aprehendido por los profesionales que actúan en el proceso de concesión del Beneficio.

Es un compromiso ético del Trabajo Social mejorar la calidad de los servicios prestados a la población. Es importante destacar que el trabajador social no es el responsable de los cambios, pero que una actuación comprometida con la garantía de los derechos de los ciudadanos puede aportar resultados significativos en relación al Beneficio de Prestación Continua.

\section{Referencias bibliográficas}

Antunes, R. (2008). Adeus ao trabalho? Ensaio sobre as metamorfoses e a centralidade do mundo do trabalho. ( $2^{\circ}$ ed.). São Paulo: Cortez.

Avila, R.I. (s/f). Os limites atuais de distribuição de renda e riqueza no Brasil. En: Carta Capital. Recuperado de: http://cartamaior.com.br/?\%2FEditoria\%2FEconomia\%2FOs-limites-atuais-da-distribuicao-de-renda-e-riqueza-no-Brasil\%2F7\%2F32456

Behring, E.R. (2008). Brasil em contra-reforma: desestruturação do Estado e perda de direitos. São Paulo, Brasil: Cortez.

Behring, E.R. y Boschetti, I. (2006). Política Social: fundamentos e história. São Paulo: Cortez.

Boschetti, I. (2004). Seguridade social e projeto ético-político: que direitos para qual cidadania? Serviço Social e Sociedade, 79, 108-132.

Boschetti, I. (2009). Seguridade social na América Latina. En: I. Boschetti. Política social no capitalismo (pp. 174-195). Brasília: Cortez Editora.

Diário Oficial da União. (1988). Constitución Federal (1988). Recuperado de: http://www.senado. gov.br/legislacao/const/con1988/con1988_05.10.1988/con1988.pdf

Diário Oficial da União. (2007) Decreto $n^{o}$ 6.214/2007. Recuperado de: http://www.planalto.gov.br/ ccivil_03/_ato2007-2010/2007/decreto/d6214.htm

Cartaxo, A.M.B. y Cabral, M.S.R. (2008). O processo de desconstrução do projeto profissional do Serviço Social na Previdência - um registro de resistência e luta dos assistentes sociais. En: L. Braga y M.S.R. Cabral (coords.), O Serviço Social na Previdência: trajetória, projetos profissionais e saberes. São Paulo: Cortez.

Conselho Federal de Serviço Social. (2001). Carta de Maceió. Recuperado de: http://www. cfess.org.br/arquivos/encontronacional_cartas_maceio.pdf

Iamamoto, M.V. (2004). O serviço social na contemporaneidade: trabalho e formação profissional. ( $7^{\circ}$ ed.) São Paulo: Cortez. 
Iamamoto, M.V. (2007). Serviço social em tempo de capital fetiche: capital financeiro, trabalho e questão social. São Paulo: Cortez.

Diário Oficial da União. (1991). Ley 8.213/1991. Recuperado de: http://www.alprevidencia.al.gov. br/legislacao/legislacoes-aplicaveis/Lei\%20n. b0\%208213-\%20de\%2025\%20de\%20Julho\% 20de\%201991\%20-\%20DOE.pdf

Diário Oficial da União Brasília. (1993). Ley 8.662/1993. Recuperado de: http://www.cfess.org.br/arquivos/CEP2011_CFESS.pdf

Diário Oficial da União. (2003). Ley 10.741/2003. Estatuto do Idoso. Recuperado de: http://www.assistenciasocial.al.gov.br/legislacao/legislacao-federal/est.\%20de\%20 idoso.pdf

Diário Oficial da União. (2010). Ley 12.317/2010. Recuperado de: http://www.cfess.org.br/arquivos/L12317.pdf

Diário Oficial da União. (2011). Ley 12.435/2011. Recuperado de: http://www.cfess.org.br/arquivos/Lei-12435.pdf

Diário Oficial da União. (2011). Ley 12.470/2011. Recuperado de: https://www.sinait.org.br/docs/ LEINo12470_DE31DEAGOSTODE_2011.pdf

Maciel, C.A.B. (2008). Beneficio de Prestação Continuada: as armadilhas. Presidente Venceslau, Brasil: Letras à margem.

Masson, G.A. (2011). Um estudo do benefício de prestação continuada no município de Altinópolis/SP: o impacto na vida de seus beneficiários (Trabajo fin de máster). Universidade Estadual Paulista, Franca, Brasil.

Netto, J. P. (2009). A construção do projeto ético-político do Serviço Social. En: A. E. Mota, (coord.), Serviço Social e Saúde: formação e trabalho social (pp. 141-160). Brasília: Ministério da Saúde.

Silva, M.L.L. (2012). Previdência Social no Brasil: (des)estruturação do trabalho e condições para sua universalização. São Paulo: Cortez.

Vaz, R.I. (2014). Acessibilidade restrita à proteção social não contributiva: um estudo sobre a invisibilidade da demanda pelo BPC. (Trabajo fin de máster). Pontifícia Universidade Católica, São Paulo.

Yazbek, M.C. (2008). Análise da Matriz Teórico-Metodológica do Serviço Social no INSS, considerando a política previdenciária, suas determinações sócio-históricas e o projeto hegemônico do Serviço Social. En: L. Braga y M.S.R. Cabral (coords.), O Serviço Social na Previdência: trajetória, projetos profissionais e saberes. São Paulo: Cortez.

Yazbek, M.C. (2009). O significado sócio-histórico da profissão. En: Serviço Social: Direitos Sociais e Competências Profissionais. Brasília: CEAD/UnB. 
